\title{
CLASSIFICATION OF THE GROUP ACTIONS ON THE REAL LINE AND CIRCLE
}

\author{
A. V. MALYUTIN
}

\begin{abstract}
The group actions on the real line and circle are classified. It is proved that each minimal continuous action of a group on the circle is either a conjugate of an isometric action, or a finite cover of a proximal action. It is also shown that each minimal continuous action of a group on the real line either is conjugate to an isometric action, or is a proximal action, or is a cover of a proximal action on the circle. As a corollary, it is proved that a continuous action of a group on the circle either has a finite orbit, or is semiconjugate to a minimal action on the circle that is either isometric or proximal. As a consequence, a new proof of the Ghys-Margulis alternative is obtained.
\end{abstract}

\section{INTRODUCTION}

In this paper, by an action of a group on a topological space we mean an action by homeomorphisms; by (semi)conjugacy of actions we mean topological (semi)conjugacy. An action of a group $G$ on a Hausdorff topological space $X$ is said to be proximal if for any two points $x$ and $y$ in $X$ there exists a sequence $\left\{g_{k}\right\}$ in $G$ such that the sequences $\left\{g_{k} x\right\}$ and $\left\{g_{k} y\right\}$ converge to one and the same point.

The key result of the paper is the following theorem (see $\S 1$ for the definitions involved).

Theorem 1. Let $X$ be either the real line $(\mathbb{R})$ or the circle $\left(\mathbf{S}^{1}\right)$. Let $\phi: G \rightarrow \operatorname{Homeo}(X)$ be a minimal action of a group $G$ on $X$ (by homeomorphisms). Then exactly one of the following three assertions is true:

(i) the action $\phi$ is conjugate to an isometric action;

(ii) the action $\phi$ is proximal;

(iii) the action $\phi$ is a nontrivial cover of a minimal and proximal action on the circle; i.e., $\phi$ is semiconjugate to a minimal and proximal action on the circle via a nontrivial covering map.

If $X=\mathbf{S}^{1}$, then assertions (ii) and (iii) can be united, and Theorem 1 takes the following form.

Theorem 1 (Case of $\mathbf{S}^{1}$ ). Every minimal continuous action of a group on the circle is either a conjugate of an isometric action or a finite cover of a proximal action.

Theorem 1 implies the following classification (see $\S 7$ for the proof).

2000 Mathematics Subject Classification. Primary 54H15; Secondary 57S25, 57M60, 54H20, 37E05, $37 \mathrm{E} 10$.

Key words and phrases. Circle, line, group of homeomorphisms, action, proximal, distal, semiconjugacy.

The author was partially supported by grant NSh-4329.2006.1 and by RFBR grant no. 05-01-00899. 
Corollary $2\left(\mathbf{S}^{1}\right)$. Let $\phi: G \rightarrow \operatorname{Homeo}\left(\mathbf{S}^{1}\right)$ be an action of a group $G$ on the circle (by homeomorphisms). Then exactly one of the following three conditions is fulfilled:

(a) the action $\phi$ has a finite orbit;

(b) the action $\phi$ is semiconjugate to an isometric minimal action on the circle via a continuous locally monotone semiconjugacy of degree 1 ;

(c) the action $\phi$ is semiconjugate to a minimal and proximal action on the circle via a continuous locally monotone semiconjugacy.

Theorem 1 also implies a similar classification (which, however, includes more cases) for the actions of groups on the real line.

A remark about alternatives. It turns out that if an action $\phi: G \rightarrow \operatorname{Homeo}\left(\mathbf{S}^{1}\right)$ meets either condition (a) or condition (b) of Corollary 2, then $\mathbf{S}^{1}$ bears a $G$-invariant Borel probability measure (this is explained in the proof of Corollary 2 in $\S 7$ ). On the other hand, it can be shown that each continuous proximal action of a group on the circle is strongly proximal in the sense of Furstenberg (see [3]; an action is strongly proximal if and only if the induced action on the associated space of Borel probability measures is proximal). Therefore, Corollary 2 implies the following alternative (where the cases are not mutually exclusive).

Every action $\phi: G \rightarrow \operatorname{Homeo}\left(\mathbf{S}^{1}\right)$ either admits an invariant Borel probability measure, or is semiconjugate to a strongly proximal action on the circle.

It is easy to show that if an action of a group $G$ on the circle is minimal and proximal, then $G$ contains a free non-Abelian subgroup (see Proposition 6 and Remark 6.2 for the proof). Therefore, Corollary 2 implies the Ghys alternative, which was proved by G. A. Margulis in [6]:

If a group $G$ acts on the circle $\mathbf{S}^{1}$ by homeomorphisms, then either there exists a $G$-invariant Borel probability measure on $\mathbf{S}^{1}$, or $G$ contains a free non-Abelian subgroup.

A remark about centralizers. Let $X$ be either the real line or the circle, and let $\phi: G \rightarrow \operatorname{Homeo}(X)$ be a minimal action (as in Theorem 1 ). Let $\mathcal{Z}$ denote the centralizer of the subgroup $\phi(G) \cap \mathrm{Homeo}_{+}(X)$ in the group Homeo $(X)$.

Assertions (i), (ii), and (iii) of Theorem 1 can be reformulated in terms of the centralizer $\mathcal{Z}$ as follows:

- (i) $\Longleftrightarrow \mathcal{Z}$ is isomorphic to the Abelian group $X$;

- (ii) $\Longleftrightarrow \mathcal{Z}$ is trivial;

- (iii) $\Longleftrightarrow \mathcal{Z}$ is a cyclic group (infinite if $X=\mathbb{R}$, and finite if $X=\mathbf{S}^{1}$ ).

We also remark that in case (iii) the orbit space $X / \mathcal{Z}$ is homeomorphic to the circle, the corresponding projection $p: X \rightarrow X / \mathcal{Z}$ is a nontrivial covering (universal if $X=\mathbb{R}$, and finite-sheeted if $X=\mathbf{S}^{1}$ ), and there exists a minimal and proximal action $\psi: G \rightarrow$ $\operatorname{Homeo}(X / \mathcal{Z})$ such that the covering $p$ is a semiconjugacy between $\phi$ and $\psi$.

On uniqueness. Let $G$ be a group, and let $X \in\left\{\mathbb{R}, \mathbf{S}^{1}\right\}$. Suppose that an action $\phi: G \rightarrow \operatorname{Homeo}(X)$ is semiconjugate to two actions $\psi_{i}: G \rightarrow \operatorname{Homeo}\left(\mathbf{S}^{1}\right)(i=1,2)$ via continuous locally monotone semiconjugacies $p_{i}: X \rightarrow \mathbf{S}^{1}(i=1,2)$, respectively. Suppose moreover that both $\psi_{1}$ and $\psi_{2}$ are minimal and proximal. Then it can be shown that $\psi_{1}$ and $\psi_{2}$ are conjugate. Furthermore, there exists a conjugacy $q: \mathbf{S}^{1} \rightarrow \mathbf{S}^{1}$ between $\psi_{1}$ and $\psi_{2}$ such that $p_{2}=q \circ p_{1}$.

It follows, in particular, that if an action $\phi: G \rightarrow \operatorname{Homeo}\left(\mathbf{S}^{1}\right)$ meets condition (c) of Corollary 2, then the absolute value of the degree of the semiconjugacy mentioned in condition (c) is an invariant of $\phi$. 
Structure of the paper. $\S 1$ contains the basic definitions.

In $\S 2$ we prove Proposition 2, which says that each distal action of a group on $X \in$ $\left\{\mathbb{R}, \mathbf{S}^{1}\right\}$ is conjugate to an isometric action.

$\S 3$ contains several lemmas involved in the proof of Proposition 4 (see $\S 4$ ).

In $\S 4$ we prove Proposition 4 , which concerns minimal group actions on the real line that are neither proximal nor distal.

In $\S 5$, we show that each minimal and nondistal action of a group on the circle is a cover of a minimal and proximal action (see Proposition 5).

In $\S 6$, we prove some properties of a minimal and proximal group action on the circle.

In $\S 7$, Theorem 1 and Corollary 2 are deduced from the results of the preceding sections.

\section{§1. Preliminaries}

1.1. Group actions on topological spaces. Let $G$ be a group, $X$ a topological space. An action of $G$ on $X$ is a homomorphism $\phi$ from $G$ to the group $\operatorname{Homeo}(X)$ of all homeomorphisms of $X$ :

$$
\phi: G \rightarrow \operatorname{Homeo}(X) .
$$

The image of a point $x \in X$ under a homeomorphism $\phi(g)(g \in G)$ is denoted by $g(x)$ or $g x$. For $F \subset G$ and $A \subset X$, we define

$$
F(A):=\{g(x) \mid g \in F, x \in A\} .
$$

For a point $x \in X$, the orbit of $x$ is the set $G(x)$. A subset $A \subset X$ is said to be $G$-invariant if $g(A)=A$ for each $g \in G$.

If $H$ is a subgroup of $\operatorname{Homeo}(X)$, then by the action of $H$ on $X$ we mean the inclusion $H \rightarrow \operatorname{Homeo}(X)$. For a given action $\phi: G \rightarrow \operatorname{Homeo}(X)$ and a subgroup $G^{\prime}$ of $G$, by the action of $G^{\prime}$ on $X$ we mean the restriction $\left.\phi\right|_{G^{\prime}}: G^{\prime} \rightarrow \operatorname{Homeo}(X)$.

An action of a group on a topological space is said to be minimal if each orbit of the action is dense (or, equivalently, if the action has no proper closed invariant subsets).

Two actions $\phi: G \rightarrow \operatorname{Homeo}(X)$ and $\psi: G \rightarrow \operatorname{Homeo}(Y)$ are conjugate if there exists a homeomorphism $f: X \rightarrow Y$ such that $g(f(x))=f(g(x))$ for any $x \in X$ and any $g \in G$. In this case, we also say that $\phi$ is conjugate to $\psi$ via $f$, and that $f$ is a conjugacy between $\phi$ and $\psi$.

An action $\phi: G \rightarrow \operatorname{Homeo}(X)$ is semiconjugate to an action $\psi: G \rightarrow \operatorname{Homeo}(Y)$ if there exists a continuous surjection $f: X \rightarrow Y$ such that $g(f(x))=f(g(x))$ for any $x \in X$ and any $g \in G$. In this case, we also say that $\phi$ is semiconjugate to $\psi$ via $f$, and that $f$ is a semiconjugacy between $\phi$ and $\psi$.

If an action $\phi$ is semiconjugate to an action $\psi$ via a covering map, we say that $\phi$ is a cover of $\psi$.

1.2. Proximal and distal actions. Let a group $G$ act on a Hausdorff topological space $X$.

Two points $x$ and $y$ in $X$ are said to be proximal with respect to the action of $G$ (or $G$-proximal) if there exists a sequence $\left\{g_{k}\right\}$ in $G$ such that the sequences $\left\{g_{k} x\right\}$ and $\left\{g_{k} y\right\}$ converge to one and the same point. If $x$ and $y$ are not $G$-proximal, they are $G$-distal.

An action of a group $G$ on a space $X$ is said to be proximal if any two points in $X$ are $G$-proximal, and it is distal if any two distinct points in $X$ are $G$-distal.

We say that a subset $A \subset X$ is $G$-contractible if there is a point $z \in X$ such that for each open neighborhood $U \ni z$ there exists an element $g \in G$ such that $g(A) \subset U$. The definitions imply immediately that any two points in a $G$-contractible subset are $G$-proximal. 
If $X$ is a metric space with a metric d, and $A$ is a subset of $X$, then we define

$$
\operatorname{diam}(A):=\sup \{\mathrm{d}(x, y) \mid x, y \in A\} .
$$

If $(X, \mathrm{~d})$ is compact, then two points $x, y \in X$ are $G$-proximal if and only if there exists a sequence $\left\{g_{k}\right\}$ in $G$ such that $\mathrm{d}\left(g_{k} x, g_{k} y\right) \rightarrow 0$. Also, if $(X, \mathrm{~d})$ is compact, a subset $A \subset X$ is $G$-contractible if and only if $G$ contains a sequence $\left\{g_{k}\right\}$ such that $\operatorname{diam}\left(g_{k} A\right) \rightarrow 0$.

1.3. Orbit spaces. For a given action of a group $T$ on a space $X$, we denote by $X / T$ the orbit space of this action (i.e., the quotient space of $X$ by the equivalence relation whose classes are the orbits of $T$ ). The map $p: X \rightarrow X / T$ that takes a point to its orbit is the quotient map. For $t \in \operatorname{Homeo}(X)$, we denote by $\langle t\rangle$ the subgroup generated by the homeomorphism $t$. In this case, we set $X / t:=X /\langle t\rangle$.

Lemma 1.3. Let $X$ be a topological space, $G$ and $T$ two subgroups of $\operatorname{Homeo}(X)$. Let $p: X \rightarrow X / T$ be the quotient map. Suppose that $G$ is contained in the normalizer of $T$ (this condition is fulfilled, in particular, if $G$ commutes with $T$ ). Then there exists an (obviously, unique) action $\varphi: G \rightarrow \operatorname{Homeo}(X / T)$ such that the action of $G$ on $X$ is semiconjugate to $\varphi$ via $p$.

Proof. Since $G$ is contained in the normalizer of $T$, it follows that for any $g \in G$ and any $x \in X$ we have

$$
g(T(x))=g\left(T\left(g^{-1} g x\right)\right)=g T g^{-1}(g x)=T(g x) .
$$

Clearly, this means that $G$ acts on the set of orbits of $T$; i.e., $G$ acts on the set of points of the orbit space $X / T$. (At this stage of the proof, we avoid saying that $G$ acts on the space $X / T$, and only say that $G$ acts on the set $X / T$, because we have not yet established the continuity of the action.)

For $g \in G$, we denote by $\varphi_{g}$ the bijection $X / T \rightarrow X / T$ that takes an orbit $T(x) \in X / T$ to the orbit $T(g x)$. Accordingly, we denote by $\varphi$ the mapping $g \mapsto \varphi_{g}$ from $G$ to the group of bijections of the set $X / T$. Then, clearly, $\varphi$ is a homomorphism. We observe that for any $g \in G$ and any $x \in X$ we have

$$
\varphi_{g}(p(x))=\varphi_{g}(T(x))=T(g x)=p(g x)
$$

by the definition of $\varphi_{g}$.

In other words,

$$
\varphi_{g} \circ p=p \circ g
$$

for each $g \in G$.

Now, we recall the universality property for quotient maps. It states that if $Y$ is a topological space, $\sim$ is an equivalence relation on $Y, Y / \sim$ is the quotient space, and $f: Y \rightarrow Y / \sim$ is the corresponding quotient map, then a map $h: Y / \sim \rightarrow Z$ to a topological space $Z$ is continuous if and only if the composition $h \circ f: Y \rightarrow Z$ is continuous.

For each element $g \in G$, the composition $\varphi_{g} \circ p$ is continuous by $(*)$. Thus, the universality property implies that $\varphi_{g}$ is continuous. This means that the bijection $\varphi_{g}$ of the set $X / T$ is indeed an autohomeomorphism of the space $X / T$. Therefore, $\varphi$ is a homomorphism from $G$ to $\operatorname{Homeo}(X / T)$. To complete the proof, we observe that, by $(*)$, the action of $G$ on $X$ is semiconjugate to $\varphi$ via $p$.

1.4. The real line and circle. As is customary, we denote by $\mathbb{R}$ the real line. A translation of $\mathbb{R}$ is a homeomorphism of the form

$$
t_{\alpha}: \mathbb{R} \rightarrow \mathbb{R}, \quad t_{\alpha}(x)=x+\alpha \quad(\alpha \in \mathbb{R}) .
$$

We define the circle $\mathbf{S}^{1}$ as the orbit space $\mathbb{R} / t_{1}$ of the translation by 1 . We denote by $\mathbf{p}: \mathbb{R} \rightarrow \mathbf{S}^{1}$ the corresponding quotient map from $\mathbb{R}$ to $\mathbb{R} / t_{1}=\mathbf{S}^{1}$. It is well known that 
$\mathbf{p}$ is a universal covering map. The group $\left\langle t_{1}\right\rangle$ of integer translations is the group of deck transformations of the covering $\mathbf{p}: \mathbb{R} \rightarrow \mathbf{S}^{1}$.

On $\mathbb{R}$, we use the standard metric $\mathrm{d}(x, y):=|x-y|$. On $\mathbf{S}^{1}$, we use the induced metric

$$
\mathrm{d}(a, b):=\min \left\{|\widetilde{a}-\widetilde{b}|: \widetilde{a} \in \mathbf{p}^{-1}(a), \widetilde{b} \in \mathbf{p}^{-1}(b)\right\} .
$$

Let $X \in\left\{\mathbb{R}, \mathbf{S}^{1}\right\}$. The group of all orientation-preserving homeomorphisms of $X$ is denoted by $\mathrm{Homeo}_{+}(X)$. For a given action $\phi: G \rightarrow \operatorname{Homeo}(X)$, we set $G_{+}:=$ $\phi^{-1}\left(\mathrm{Homeo}_{+}(X)\right)$. Clearly, $G_{+}$is a normal subgroup in $G$ of index at most 2 .

As usual, we denote by $\widetilde{\operatorname{Homeo}}\left(\mathbf{S}^{1}\right)$ the subgroup of $\operatorname{Homeo}(\mathbb{R})$ that consists of all lifts of all homeomorphisms in Homeo $\left(\mathbf{S}^{1}\right)$. Accordingly, the group Homeo $\left(\mathbf{S}^{1}\right) \cap \mathrm{Homeo}_{+}(\mathbb{R})$ is denoted by $\mathrm{Homeo}_{+}\left(\mathbf{S}^{1}\right)$. In the group Homeo $(\mathbb{R})$, the subgroup Homeo $\left(\mathbf{S}^{1}\right)$ is the normalizer of the subgroup $\left\langle t_{1}\right\rangle$ of integer translations, while Homeo $+\left(\mathbf{S}^{1}\right)$ is the centralizer of $\left\langle t_{1}\right\rangle$.

We denote by $\mathbf{p}_{*}$ the natural homomorphism from $\operatorname{Homeo}\left(\mathbf{S}^{1}\right)$ to Homeo $\left(\mathbf{S}^{1}\right)$ :

$$
\mathbf{p}_{*}: \text { Homeo }\left(\mathbf{S}^{1}\right) \rightarrow \operatorname{Homeo}\left(\mathbf{S}^{1}\right) \text {. }
$$

Since the covering $\mathbf{p}: \mathbb{R} \rightarrow \mathbf{S}^{1}$ is universal, each homeomorphism of the circle has lifts to $\mathbb{R}$. In other words, $\mathbf{p}_{*}$ is an epimorphism. The group $\left\langle t_{1}\right\rangle$ is the kernel of $\mathbf{p}_{*}$. Thus, we have the following exact sequence:

$$
0 \rightarrow\left\langle t_{1}\right\rangle \rightarrow \text { Homeo }\left(\mathbf{S}^{1}\right) \rightarrow \operatorname{Homeo}\left(\mathbf{S}^{1}\right) \rightarrow 1 .
$$

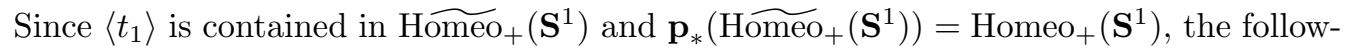
ing sequence is also exact:

$$
0 \rightarrow\left\langle t_{1}\right\rangle \rightarrow \mathrm{Homeo}_{+}\left(\mathbf{S}^{1}\right) \rightarrow \mathrm{Homeo}_{+}\left(\mathbf{S}^{1}\right) \rightarrow 1 .
$$

Lemma 1.4. Suppose that a homeomorphism $h \in \operatorname{Homeo}(\mathbb{R})$ has no fixed points. Then $h$ is conjugate to the translation $t_{1}$. Consequently, the orbit space $\mathbb{R} / h$ is homeomorphic to the circle, and the corresponding quotient map $\mathbb{R} \rightarrow \mathbb{R} / h$ is a universal covering.

Proof. We define a map

$$
F_{h}: \mathbb{R} \rightarrow \mathbb{R}, \quad F_{h}(x):=h^{\lfloor x\rfloor}(h(0) \cdot\{x\}),
$$

where $\lfloor x\rfloor$ is the integral part of $x$, and $\{x\}=x-\lfloor x\rfloor$.

To show that the map $F_{h}$ is continuous, we observe that for each integer $k$ the map $F_{h}$ takes the form $h^{k}(h(0) \cdot(x-k))$ on the half-open interval $[k, k+1)$. Therefore, $F_{h}$ is continuous on $[k, k+1)$. It remains to check that $F_{h}$ is left-continuous at the integer points. Let $k \in \mathbb{Z}$, and let $x \rightarrow k^{-}$(i.e., $x \rightarrow k$ and $x<k$ ). Then $\{x\} \rightarrow 1$. Since $F_{h}(k)=h^{k}(h(0) \cdot 0)=h^{k}(0)$, we have

$$
\lim _{x \rightarrow k^{-}} F_{h}(x)=h^{k-1}\left(h(0) \cdot \lim _{x \rightarrow k^{-}}\{x\}\right)=h^{k-1}(h(0))=h^{k}(0)=F_{h}(k) .
$$

So, indeed, $F_{h}$ is continuous.

Next, we show that $F_{h}$ is strictly monotone. For each $k \in \mathbb{Z}$, the function $\{x\}$ is monotone increasing on the half-open interval $[k, k+1)$. Consequently, if $h(0)>0$, then the function $h(0) \cdot\{x\}$ increases on $[k, k+1)$, and if $h(0)<0$, then $h(0) \cdot\{x\}$ decreases on $[k, k+1)$. Note that $h(0) \neq 0$ because $h$ has no fixed points. Combining the assumption that $h$ has no fixed points and the obvious fact that each orientationreversing homeomorphism of the real line has a (unique) fixed point, we see that $h$ preserves orientation. Therefore, the map $h^{k}$ is monotone increasing for each integer $k$. The above implies that the map $F_{h}$ increases on an interval $[k, k+1)$ if $h(0)>0$, and it decreases if $h(0)<0$. Then the continuity of $F_{h}$ implies that $F_{h}$ is strictly monotone.

Finally, we observe that since $h$ has no fixed points, the set $\left\{F_{h}(k)=h^{k}(0)\right\}_{k \in \mathbb{Z}}$ is unbounded (both from above and from below). 
The map $F_{h}$ is thus continuous, strictly monotone, and unbounded from above and from below. Consequently, $F_{h}$ is a homeomorphism of the real line.

Furthermore, the definition of $F_{h}$ implies that

$$
F_{h}(x+1)=h^{\lfloor x+1\rfloor}(h(0) \cdot\{x+1\})=h h^{\lfloor x\rfloor}(h(0) \cdot\{x\})=h F_{h}(x) .
$$

In other words, we have $F_{h} t_{1}=h F_{h}$, whence

$$
F_{h}^{-1} h F_{h}=t_{1} .
$$

So, $h$ is conjugate to $t_{1}$ via the homeomorphism $F_{h}^{-1}$.

Maps of the circle. Let $f: \mathbf{S}^{1} \rightarrow \mathbf{S}^{1}$ be a continuous map, and let $\tilde{f}: \mathbb{R} \rightarrow \mathbb{R}$ be an arbitrary lift of $f$ to the universal cover $\mathbb{R}$.

The difference $\tilde{f}(x+1)-\tilde{f}(x)$ is an integer, which depends neither on the choice of the lift $\tilde{f}$, nor on the choice of the point $x \in \mathbb{R}$. The number $\tilde{f}(x+1)-\tilde{f}(x)$ is called the degree of $f$. (This is the customary Brouwer degree in the case of $\mathbf{S}^{1}$.)

The map $f$ is said to be locally monotone if $\tilde{f}$ is monotone. (Obviously, the monotonicity of a lift does not depend on the choice of the lift.)

\section{$\S 2$. Distal actions}

In this section, we prove the following proposition.

Proposition 2. Let $X$ be either the real line $\mathbb{R}$ or the circle $\mathbf{S}^{1}$. Then each distal action $\phi: G \rightarrow \operatorname{Homeo}(X)$ is conjugate to an isometric action.

The rest of this section is devoted to the proof.

Proof in the case where $X=\mathbf{S}^{1}$. We recall that an action of a group $H$ on a metric space $(M, \mathrm{~d})$ is equicontinuous if for each $\varepsilon>0$ there exists $\delta(\varepsilon)>0$ such that for each $h \in H$ and any $x, y \in M$ with $\mathrm{d}(x, y)<\delta(\varepsilon)$ we have $\mathrm{d}(h x, h y)<\varepsilon$.

It is known that each equicontinuous action of a group on the circle is conjugate to an isometric action (see, e.g., [6, Lemma 3]). Therefore, in our case it suffices to show that the action $\phi: G \rightarrow \operatorname{Homeo}\left(\mathbf{S}^{1}\right)$ is equicontinuous.

Claim 2.1. If the action $\phi: G \rightarrow \operatorname{Homeo}\left(\mathbf{S}^{1}\right)$ is distal, then it is equicontinuous.

Proof. Assume the contrary, i.e., let $\phi$ be distal but not equicontinuous. Then by definition there exists a number $\varepsilon>0$ and three sequences

$$
\left\{g_{k}\right\} \text { in } G \text { and }\left\{x_{k}\right\},\left\{y_{k}\right\} \text { in } \mathbf{S}^{1}
$$

such that

$$
\mathrm{d}\left(x_{k}, y_{k}\right)<\frac{1}{k} \quad \text { and } \quad \mathrm{d}\left(g_{k}\left(x_{k}\right), g_{k}\left(y_{k}\right)\right) \geq \varepsilon .
$$

It follows that there exists a sequence $\left\{I_{k}\right\}$ of closed intervals on the circle such that

$$
\operatorname{diam}\left(I_{k}\right)<\frac{1}{k} \quad \text { and } \quad \operatorname{diam}\left(g_{k} I_{k}\right) \geq \varepsilon
$$

Since $\mathbf{S}^{1}$ is compact, there is an infinite subsequence $\left\{g_{k_{i}} I_{k_{i}}\right\}$ in $\left\{g_{k} I_{k}\right\}$ and a nondegenerate interval $I \subset \mathbf{S}^{1}$ such that

Then $I_{k_{i}} \supset g_{k_{i}}^{-1}(I)$, whence it follows that

$$
g_{k_{i}} I_{k_{i}} \supset I \text {. }
$$

$$
\operatorname{diam}\left(g_{k_{i}}^{-1}(I)\right)<\frac{1}{k_{i}} .
$$

Thus, we have proved that the interval $I$ is $G$-contractible; hence, any two points in $I$ are $G$-proximal. This means that $\phi$ is not distal, a contradiction. 
Proof in the case where $X=\mathbb{R}$. Let $H_{+}$denote the subgroup $\phi(G) \cap \mathrm{Homeo}_{+}(\mathbb{R})$. Obviously, the distality of $\phi$ implies that the action of $H_{+}$is also distal.

Claim 2.2. The subgroup $H_{+}$acts freely on $\mathbb{R}$, i.e., each nontrivial homeomorphism in $H_{+}$has no fixed points.

Proof. Assume that $H_{+}$contains a nontrivial homeomorphism $h$ that has a fixed point. Let $h(x)=x$ and $h(y) \neq y$. We observe that, since $h$ preserves orientation, the two-sided sequence $\left\{h^{i}(y)\right\}_{i \in \mathbb{Z}}$ is monotone, and all points of the sequence are contained in one and the same component of $\mathbb{R} \backslash x$. Hence, at least one of the two sequences $\left\{h^{k}(y)\right\}_{k \in \mathbb{N}}$ and $\left\{h^{-k}(y)\right\}_{k \in \mathbb{N}}$ converges. Let $g \in\left\{h, h^{-1}\right\}$, and let the sequence $\left\{g^{k}(y)\right\}_{k \in \mathbb{N}}$ converge. Then it is clear that the sequence $\left\{g^{k}(h y)\right\}_{k \in \mathbb{N}}$ has the same limit point as $\left\{g^{k}(y)\right\}_{k \in \mathbb{N}}$. This means that the points $y$ and $h(y)$ are $H_{+}$-proximal, which contradicts our assumptions.

Lemma 2.1. Let $\mathcal{H}$ be a subgroup of $\operatorname{Homeo}\left(\mathbf{S}^{1}\right)$, and let $\widetilde{\mathcal{H}}:=\mathbf{p}_{*}^{-1}(\mathcal{H})$ denote the subgroup of $\mathrm{Homeo}\left(\mathbf{S}^{1}\right)$ that consists of all lifts of homeomorphisms in $\mathcal{H}$. Suppose that the action of $\mathcal{H}$ on $\mathbf{S}^{1}$ is not distal. Then the action of $\widetilde{\mathcal{H}}$ on $\mathbb{R}$ is not distal.

Proof. Since the action of $\mathcal{H}$ on $\mathbf{S}^{1}$ is not distal, it follows that there are two points $x \neq y \in \mathbf{S}^{1}$ that are $\mathcal{H}$-proximal. This easily implies that at least one of the two open intervals that compose the space $\mathbf{S}^{1} \backslash\{x, y\}$ (say, an interval $I$ ) is $\mathcal{H}$-contractible. Therefore, there exists a sequence $\left\{h_{k}\right\}$ in $\mathcal{H}$ such that $\operatorname{diam} h_{k}(I) \rightarrow 0$. Let $\widetilde{I} \subset \mathbb{R}$ be an interval (a component) of the inverse image $\mathbf{p}^{-1}(I)$. We recall that the kernel of the homomorphism $\mathbf{p}_{*}:$ Homeo $\left(\mathbf{S}^{1}\right) \rightarrow \operatorname{Homeo}\left(\mathbf{S}^{1}\right)$ is the group $\left\langle t_{1}\right\rangle$ of integer translations, and for an arbitrary homeomorphism $h \in \operatorname{Homeo}\left(\mathbf{S}^{1}\right)$ the inverse image $\mathbf{p}_{*}^{-1}(h)$ has the form $\left\langle t_{1}\right\rangle \widetilde{h}_{0}$, where $\widetilde{h}_{0}$ is any lift of $h$. Now, $\operatorname{since} \operatorname{diam}(\widetilde{h}(\widetilde{I})) \leq 1$ for each $\widetilde{h} \in \operatorname{Homeo}\left(\mathbf{S}^{1}\right)$, it follows that for each $h_{k}$ there exists a lift $\widetilde{h}_{k}$ such that $\widetilde{h}_{k}(\widetilde{I}) \subset[0,2]$. Observe that $\operatorname{diam} \widetilde{h}_{k}(\widetilde{I}) \rightarrow 0$ (because $\operatorname{diam} h_{k}(I) \rightarrow 0$ ). Then the compactness of the segment $[0,2]$ implies that the interval $\widetilde{I}$ is $\widetilde{\mathcal{H}}$-contractible. Consequently, any two points in $\widetilde{I}$ are $\widetilde{\mathcal{H}}$-proximal. Then the action of $\widetilde{\mathcal{H}}$ on $\mathbb{R}$ is not distal, as required.

Claim 2.3. The subgroup $H_{+}$is conjugate to a subgroup consisting of translations.

Proof. By Hölder's theorem, if a group acts freely on the real line by homeomorphisms, then this group is Abelian, and so Claim 2.2 implies that $H_{+}$is Abelian. If $H_{+}$is trivial, then Claim 2.3 is obviously true. Assume that $H_{+}$is nontrivial. Let $t \in H_{+}$be a nontrivial element. By Claim 2.2, $t$ has no fixed points. Then Lemma 1.4 yields the existence of $f_{1} \in \operatorname{Homeo}(\mathbb{R})$ such that $f_{1} t f_{1}^{-1}=t_{1}$. Thus, the group $f_{1} H_{+} f_{1}^{-1}$ is Abelian and contains the translation by 1 (which we denote by $t_{1}$ ). In particular, $f_{1} H_{+} f_{1}^{-1}$ commutes with $t_{1}$, whence we conclude that $f_{1} H_{+} f_{1}^{-1}$ is contained in $\widehat{\mathrm{Homeo}}+\left(\mathbf{S}^{1}\right)$. Consider the subgroup $F:=\mathbf{p}_{*}\left(f_{1} H_{+} f_{1}^{-1}\right)$ of Homeo+ $\left(\mathbf{S}^{1}\right)$ (see Subsection 1.4 for the definition of $\mathbf{p}_{*}$ ). We observe that the action of $f_{1} H_{+} f_{1}^{-1}$ on $\mathbb{R}$ is distal (because the action of $H_{+}$is distal) and that $f_{1} H_{+} f_{1}^{-1}=\mathbf{p}_{*}^{-1}(F)$ (because $t_{1} \in f_{1} H_{+} f_{1}^{-1}$ ). Combining these facts with Lemma 2.1, we see that the action of $F$ on the circle is distal. Therefore, by the first part of the proposition (we mean the case where $X=\mathbf{S}^{1}$ ), the action of $F$ on $\mathbf{S}^{1}$ is conjugate to an isometric action. Moreover, since $F \subset \mathrm{Homeo}_{+}\left(\mathbf{S}^{1}\right)$, it follows that for some $f_{2} \in \operatorname{Homeo}\left(\mathbf{S}^{1}\right)$ the subgroup $f_{2} F f_{2}^{-1}$ consists of rotations. Let $\widetilde{f}_{2}$ be a lift of $f_{2}$. Then

$$
f_{2} F f_{2}^{-1}=\mathbf{p}_{*}\left(\widetilde{f}_{2} f_{1} H_{+} f_{1}^{-1} \tilde{f}_{2}^{-1}\right) .
$$

Now, since $\mathbf{p}_{*}\left(\widetilde{f}_{2} f_{1} H_{+} f_{1}^{-1} \widetilde{f}_{2}^{-1}\right)$ consists of rotations, it follows that $\widetilde{f}_{2} f_{1} H_{+} f_{1}^{-1} \widetilde{f}_{2}^{-1}$ consists of translations. 
Lemma 2.2. Let $\mathcal{H}$ be a subgroup of $\operatorname{Homeo}(\mathbb{R})$. Suppose that the subgroup $\mathcal{H}_{+}:=$ $\mathcal{H} \cap \mathrm{HomeO}_{+}(\mathbb{R})$ consists of translations. Then the subgroup $\mathcal{H}$ is conjugate to a subgroup consisting of isometries.

Proof. Since the case where $\mathcal{H}=\mathcal{H}_{+}$is trivial, we assume that $\mathcal{H} \neq \mathcal{H}_{+}$. Then $\mathcal{H}_{+}$is a subgroup of index 2 in $\mathcal{H}$. We fix an arbitrary element $h \in \mathcal{H} \backslash \mathcal{H}_{+}$and consider the map $f$ from $\mathbb{R}$ to $\mathbb{R}$ defined by

$$
f(x):=x-h(x) .
$$

The map $f$ is a homeomorphism. (To check this, observe that the map $h: \mathbb{R} \rightarrow \mathbb{R}$ is continuous and monotone decreasing. Hence, the map $-h: \mathbb{R} \rightarrow \mathbb{R}$ is continuous and monotone increasing, which easily implies the statement.)

Let us show that the group $f \mathcal{H} f^{-1}$ consists of isometries.

First, let $g$ be an element of $\mathcal{H}_{+}$. Then for some $\alpha \in \mathbb{R}$ we have $g(x)=x+\alpha$. Observe that $h g h^{-1} \in \mathcal{H}_{+}$. Consequently, $h g h^{-1}(x)=x+\beta$ for some $\beta \in \mathbb{R}$. Therefore, we obtain

$$
f g(x)=g(x)-h g(x)=g(x)-h g h^{-1} h(x)=x+\alpha-(h(x)+\beta)=f(x)+(\alpha-\beta) .
$$

Then

$$
f g f^{-1}(x)=f\left(f^{-1}(x)\right)+(\alpha-\beta)=x+(\alpha-\beta) .
$$

Thus, $f g f^{-1}$ is the translation by $\alpha-\beta$. In particular, $f g f^{-1}$ is an isometry.

Now, let $v$ be an element in $\mathcal{H} \backslash \mathcal{H}_{+}$. Then $h v$ and $v h^{-1}$ lie in $\mathcal{H}_{+}$. Consequently, for some $\alpha, \beta \in \mathbb{R}$ we have $h v(x)=x+\alpha$ and $v h^{-1}(x)=x+\beta$. Therefore,

$$
f v(x)=v(x)-h v(x)=v h^{-1} h(x)-(x+\alpha)=h(x)+\beta-x-\alpha=-f(x)+(\beta-\alpha) .
$$

Then

$$
f v f^{-1}(x)=-f\left(f^{-1}(x)\right)+(\beta-\alpha)=-x+(\beta-\alpha) .
$$

Thus, $f v f^{-1}$ is an isometry, being the composition of the isometry $x \mapsto-x$ with the translation by $\beta-\alpha$.

Now, the proof in the case where $X=\mathbb{R}$ follows from Claim 2.3 and Lemma 2.2.

\section{§3. Several Lemmas}

Here we prove lemmas involved in the proof of Proposition 4 (see $\S 4$ ).

Lemma 3.1. Let a group $\mathcal{G}$ act on a Hausdorff topological space $\mathcal{X}$, and let $\mathcal{G}^{\prime}$ be a subgroup of finite index in $\mathcal{G}$. Then two points $x, y \in \mathcal{X}$ are $\mathcal{G}$-proximal if and only if they are $\mathcal{G}^{\prime}$-proximal.

Proof. If $x$ and $y$ are $\mathcal{G}^{\prime}$-proximal, then their $\mathcal{G}$-proximality is an immediate consequence of the definitions. Conversely, suppose that $x$ and $y$ are $\mathcal{G}$-proximal. Then, by definition, there exists a sequence $\left\{g_{k}\right\}$ in $\mathcal{G}$ such that the sequences $\left\{g_{k} x\right\}$ and $\left\{g_{k} y\right\}$ converge to a point $z \in \mathcal{X}$. Since $\mathcal{G}^{\prime}$ has finite index, it follows that $\left\{g_{k}\right\}$ has an infinite subsequence $\left\{g_{k_{i}}\right\}$ contained in a single coset $h \mathcal{G}^{\prime}$ for some $h \in \mathcal{G}$. The sequence $\left\{h^{-1} g_{k_{i}}\right\}$ consists of elements of the subgroup $\mathcal{G}^{\prime}$, and the sequences $\left\{h^{-1} g_{k_{i}} x\right\}$ and $\left\{h^{-1} g_{k_{i}} y\right\}$ converge to one and the same point $h^{-1} z$. This means that $x$ and $y$ are $\mathcal{G}^{\prime}$-proximal, as required.

Lemma 3.2. Let a group $\mathcal{G}$ act on a connected topological space $\mathcal{X}$, and let $\mathcal{G}^{\prime}$ be a subgroup of finite index in $\mathcal{G}$. Suppose that the action of $\mathcal{G}$ is minimal. Then the action of $\mathcal{G}^{\prime}$ is also minimal. 
Proof. Assume that the action of $\mathcal{G}^{\prime}$ is not minimal, i.e., there exists a proper closed $\mathcal{G}^{\prime}$-invariant subset $K \subset \mathcal{X}$. Then the boundary $\partial K:=K \backslash \operatorname{int}(K)$ is also closed and $\mathcal{G}^{\prime}$-invariant. Moreover, since $K$ is proper and $\mathcal{X}$ is connected, it follows that $\partial K$ is nonempty.

We recall that a subset of a topological space is nowhere dense if its closure has empty interior. It easily follows that the union of a finite number of nowhere dense subsets is nowhere dense. The definitions also imply that the boundary $\partial L$ of every subset $L \subset \mathcal{X}$ is a nowhere dense subset.

We consider the set $\mathcal{G}(\partial K)$. Clearly, this set is $\mathcal{G}$-invariant. Furthermore, since $\partial K$ is nonempty, $\mathcal{G}(\partial K)$ is also nonempty. Let $h_{1}, \ldots, h_{n}$ be a set of right coset representatives of $\mathcal{G}^{\prime}$ in $\mathcal{G}$. Then $\mathcal{G}=h_{1} \mathcal{G}^{\prime} \cup \ldots \cup h_{n} \mathcal{G}^{\prime}$. Therefore, since $\mathcal{G}^{\prime}(\partial K)=\partial K$, we have

$$
\mathcal{G}(\partial K)=h_{1} \mathcal{G}^{\prime}(\partial K) \cup \ldots \cup h_{n} \mathcal{G}^{\prime}(\partial K)=h_{1}(\partial K) \cup \ldots \cup h_{n}(\partial K) .
$$

We observe that each set $h_{i}(\partial K)$ is closed and nowhere dense because so is $\partial K$. Thus, the set $\mathcal{G}(\partial K)$ is a union of finitely many nowhere dense subsets. Consequently, $\mathcal{G}(\partial K)$ is also closed and nowhere dense. In particular, we have $\mathcal{G}(\partial K) \neq \mathcal{X}$.

We have proved that $\mathcal{G}(\partial K)$ is a proper closed $\mathcal{G}$-invariant subset of $\mathcal{X}$. This means that the action of $\mathcal{G}$ is not minimal, which contradicts our assumption.

Lemma 3.3. Let a group $\mathcal{G}$ act on $\mathbb{R}$ by homeomorphisms. Suppose that the endpoints of the interval $I \subset \mathbb{R}$ are $\mathcal{G}$-proximal. Then $I$ is $\mathcal{G}$-contractible. (In particular, any two points in $I$ are $\mathcal{G}$-proximal.)

Proof. This follows easily from the definitions.

Lemma 3.4. Let a group $\mathcal{G}$ act on a Hausdorff topological space $\mathcal{X}$ by homeomorphisms. Suppose that this action is minimal. Let $\tau: \mathcal{X} \rightarrow \mathcal{X}$ be a continuous map. Suppose that $\tau$ is not an identity and commutes with the action of $\mathcal{G}$. Then:

1) the map $\tau$ has no fixed points;

2) furthermore, for each $x \in \mathcal{X}$ the points $x$ and $\tau(x)$ are $\mathcal{G}$-distal.

Proof. 1) Assume that $\tau(x)=x$ for some $x \in \mathcal{X}$. Then for each $g \in \mathcal{G}$ we have $\tau(g(x))=$ $g(\tau(x))=g(x)$. In other words, the orbit $\mathcal{G}(x)$ consists of fixed points of the map $\tau$. Since the action is minimal, it follows that $\mathcal{G}(x)$ is dense. Thus, the set of fixed points of the continuous map $\tau$ is dense. Since $\mathcal{X}$ is a Hausdorff space, it follows that $\tau$ is the identity, which gives a contradiction.

2) Assume that for a point $x \in \mathcal{X}$ the points $x$ and $\tau(x)$ are $\mathcal{G}$-proximal. Then, by definition, there exists a sequence $\left\{g_{k}\right\}$ in $\mathcal{G}$ such that the sequences $\left\{g_{k}(x)\right\}$ and $\left\{g_{k}(\tau(x))\right\}$ converge to one and the same point, say $z$. Since $\tau$ commutes with the action of $\mathcal{G}$, we have $g_{k}(\tau(x))=\tau\left(g_{k}(x)\right)$, whence

$$
z=\lim _{k \rightarrow+\infty} g_{k}(\tau(x))=\lim _{k \rightarrow+\infty} \tau\left(g_{k}(x)\right)=\tau\left(\lim _{k \rightarrow+\infty} g_{k}(x)\right)=\tau(z) .
$$

Therefore, $z$ is a fixed point of $\tau$. This contradicts the assertion of the first part of the lemma.

The proof of Proposition 4 below also involves the following well-known fact.

Lemma 3.5. Let $\mathcal{A}$ be a group, $\mathcal{B}$ a subgroup of $\mathcal{A}$. Let $\mathcal{Z}$ and $\mathcal{N}$ denote the centralizer and the normalizer of $\mathcal{B}$ in $\mathcal{A}$, respectively. Then $\mathcal{N}$ is contained in the normalizer $\mathcal{N}(\mathcal{Z})$ of $\mathcal{Z}$ in $\mathcal{A}$.

Proof. Obviously, it suffices to show that for any elements $h \in \mathcal{N}$ and $z \in \mathcal{Z}$ the element $h z h^{-1}$ is in $\mathcal{Z}$. Let $b$ be an element in $\mathcal{B}$. Then $h^{-1} b h$ is also in $\mathcal{B}$. Consequently, $h^{-1} b h$ 
commutes with $z$. This yields

$$
h z h^{-1} b=h z\left(h^{-1} b h\right) h^{-1}=h\left(h^{-1} b h\right) z h^{-1}=b h z h^{-1} .
$$

Therefore, $h z h^{-1}$ indeed commutes with each element $b \in \mathcal{B}$, i.e., we have $h z h^{-1} \in \mathcal{Z}$.

\section{$\S 4$. Minimal group aCtions on $\mathbb{R}$ \\ THAT ARE NEITHER PROXIMAL NOR DISTAL}

Proposition 4. Let $\phi: G \rightarrow \operatorname{Homeo}(\mathbb{R})$ be an action of a group $G$ on $\mathbb{R}$ by homeomorphisms. Suppose that $\phi$ is minimal and neither proximal nor distal. Then the following assertions are true.

1) For each point $x \in \mathbb{R}$, the set $\operatorname{Prox}_{\phi}(x)$ of points in $\mathbb{R}$ that are $\phi$-proximal to $x$ is an open bounded interval.

2) The map $\tau_{\phi}: \mathbb{R} \rightarrow \mathbb{R}$ defined by $\tau_{\phi}(x):=\sup \operatorname{Prox}_{\phi}(x)$ is a fixed point free homeomorphism.

3) The orbit space $\mathbb{R} / \tau_{\phi}$ is homeomorphic to the circle, and the corresponding quotient map $\mathbf{p}_{\tau_{\phi}}: \mathbb{R} \rightarrow \mathbb{R} / \tau_{\phi}$ is a universal covering.

4) The homeomorphism $\tau_{\phi}$ commutes with the subgroup $\phi\left(G_{+}\right)$(recall that $G_{+} \stackrel{\text { def }}{=}$ $\left.\phi^{-1}\left(\mathrm{Homeo}_{+}(\mathbb{R})\right)\right)$. Moreover, the subgroup $\left\langle\tau_{\phi}\right\rangle$ generated by $\tau_{\phi}$ is the centralizer of the subgroup $\phi\left(G_{+}\right)$in the group Homeo $(\mathbb{R})$.

5) The subgroup $\phi(G)$ is contained in the normalizer of $\left\langle\tau_{\phi}\right\rangle$. There exists a (unique) action $\psi: G \rightarrow \operatorname{Homeo}\left(\mathbb{R} / \tau_{\phi}\right)$ such that $\phi$ is semiconjugate to $\psi$ via $\mathbf{p}_{\tau_{\phi}}$.

6) The action $\psi$ is minimal and proximal.

Remark 4. Under the assumptions of Proposition $4, G_{+}$is a subgroup in $G$ of index at most 2. Hence, by Lemma 3.2, the action of $G_{+}$on $\mathbb{R}$ is minimal, while Lemma 3.1 implies that two points in $\mathbb{R}$ are $G$-proximal if and only if they are $G_{+}$-proximal.

Proof of 1). First, we observe that the set $\operatorname{Prox}_{\phi}(x)$ is connected. This follows from Lemma 3.3, which implies that for a point $y \in \operatorname{Prox}_{\phi}(x)$ the interval with endpoints $x$ and $y$ is contained in $\operatorname{Prox}_{\phi}(x)$.

Next, we show that the set $\operatorname{Prox}_{\phi}(x)$ is bounded. Since the action $\phi$ is not proximal, there are $G$-distal points $a<b$ in $\mathbb{R}$. Since the action of $G_{+}$is minimal (see Remark 4 ), there exists $g \in G_{+}$such that $x<g(a)$. Observe that the points $g(a)$ and $g(b)$ are $G$ distal (because $a$ and $b$ are $G$-distal), and that $g(a)<g(b)$ (because the homeomorphism $\phi(g)$ is in $\mathrm{Homeo}_{+}(\mathbb{R})$, so that it preserves orientation). The fact that $g(a)$ and $g(b)$ are $G$-distal, the condition $x<g(a)<g(b)$, and Lemma 3.3 imply that $x$ and $g(b)$ are also $G$-distal, i.e., $g(b) \notin \operatorname{Prox}_{\phi}(x)$. Thus, $g(b) \notin \operatorname{Prox}_{\phi}(x), x \in \operatorname{Prox}_{\phi}(x)$, and $x<g(b)$. Since $\operatorname{Prox}_{\phi}(x)$ is connected, these conditions imply that $\operatorname{Prox}_{\phi}(x)$ is bounded from above by $g(b)$. A similar argument shows that $\operatorname{Prox}_{\phi}(x)$ is bounded from below.

Finally, we show that the set $\operatorname{Prox}_{\phi}(x)$ is open. Let $y$ be a point in $\operatorname{Prox}_{\phi}(x)$ (the case $y=x$ is allowed). Since $\phi$ is not distal, it follows that $\mathbb{R}$ contains two distinct $G$-proximal points. Then Lemma 3.3 shows that $\mathbb{R}$ contains a nondegenerate $G$-contractible interval $I$. Since $x$ and $y$ are proximal and $\phi$ is minimal, it follows that there is an element $g \in G$ such that $g(x)$ and $g(y)$ lie in $\operatorname{int}(I)$. We explain this implication in detail. The definition of proximality implies the existence of a sequence $\left\{g_{k}\right\}$ in $G$ such that the sequences $\left\{g_{k}(x)\right\}$ and $\left\{g_{k}(y)\right\}$ converge to a point $z \in \mathbb{R}$. Since $\phi$ is minimal, it follows that there is an element $h \in G$ such that $h(z) \in \operatorname{int}(I)$. Then the sequences $\left\{h g_{k}(x)\right\}$ and $\left\{h g_{k}(y)\right\}$ converge to $h(z)$. Consequently, for some $n \in \mathbb{N}$ the points $h g_{n}(x)$ and $h g_{n}(y)$ lie in $\operatorname{int}(I)$. We set $g:=h g_{n}$.

Then the points $x$ and $y$ lie in the open interval $J:=g^{-1}(\operatorname{int} I)$. Clearly, since int $(I)$ is $G$-contractible, so is the interval $J$. By the definition of $G$-contractibility, the condition 
$x \in J$ implies that $J \subset \operatorname{Prox}_{\phi}(x)$. Thus, we have shown that an arbitrary point $(y)$ in $\operatorname{Prox}_{\phi}(x)$ has an open neighborhood $(J \ni y)$ contained in $\operatorname{Prox}_{\phi}(x)$. Consequently, the set $\operatorname{Prox}_{\phi}(x)$ is open.

We have proved that the set $\operatorname{Prox}_{\phi}(x)$ is connected, bounded, and open. Consequently, $\operatorname{Prox}_{\phi}(x)$ is an open bounded interval, as required.

To prove assertion 2), we need several auxiliary statements. (In these statements, we assume the conditions of Proposition 4.)

Claim 4.1. The map

$$
\tau_{\phi}(x):=\sup \operatorname{Prox}_{\phi}(x)
$$

commutes with the action of $G_{+}$. In other words, for each $g$ in $G_{+}$and each $x$ in $\mathbb{R}$ we have

$$
g\left(\tau_{\phi}(x)\right)=\tau_{\phi}(g(x)) .
$$

Proof. By definition, for any $x \in \mathbb{R}$ and any $g \in G$ we have

$$
g\left(\operatorname{Prox}_{\phi}(x)\right)=\operatorname{Prox}_{\phi}(g(x)) .
$$

It follows that if $g \in G_{+}$(i.e., if the homeomorphism $\phi(g)$ preserves orientation), then

$$
g\left(\sup \operatorname{Prox}_{\phi}(x)\right)=\sup g\left(\operatorname{Prox}_{\phi}(x)\right) \text {. }
$$

Therefore, for any $g \in G_{+}$and any $x \in \mathbb{R}$ we have

$$
\begin{aligned}
g\left(\tau_{\phi}(x)\right) & \stackrel{\operatorname{def} \tau_{\phi}}{=} g\left(\sup \operatorname{Prox}_{\phi}(x)\right) \\
& \stackrel{(2)}{=} \sup g\left(\operatorname{Prox}_{\phi}(x)\right) \stackrel{(1)}{=} \sup \operatorname{Prox}_{\phi}(g(x)) \stackrel{\operatorname{def} \tau_{\phi}}{=} \tau_{\phi}(g(x)) .
\end{aligned}
$$

Claim 4.2. For each $x \in \mathbb{R}$, we have $\tau_{\phi}(x)>x$.

Proof. It immediately follows from the definition that $x \in \operatorname{Prox}_{\phi}(x)$. By assertion 1) of the proposition, the set $\operatorname{Prox}_{\phi}(x)$ is open. Consequently,

$$
\tau_{\phi}(x)=\sup \operatorname{Prox}_{\phi}(x)>x .
$$

Claim 4.3. The map $\tau_{\phi}$ is monotone nondecreasing. In other words, if $x<y \in \mathbb{R}$, then $\tau_{\phi}(x) \leq \tau_{\phi}(y)$.

Proof. Assume that $x<y \in \mathbb{R}$ and $\tau_{\phi}(x)>\tau_{\phi}(y)$. Then Claim 4.2 implies that

$$
x<y<\tau_{\phi}(y)<\tau_{\phi}(x)
$$

Combining this with the definition of $\tau_{\phi}$ and also with assertion 1) of the proposition, we conclude that $\tau_{\phi}(y)$ lies in the set $\operatorname{Prox}_{\phi}(x)$, i.e., $x$ and $\tau_{\phi}(y)$ are $G$-proximal. Then, by Lemma 3.3 , the points $y$ and $\tau_{\phi}(y)$ are also $G$-proximal. By the definition of $\tau_{\phi}$, this contradicts assertion 1 ) of the proposition. Hence, $\tau_{\phi}(x) \leq \tau_{\phi}(y)$.

Claim 4.4. The map $\tau_{\phi}$ is continuous and surjective.

Proof. We observe that the image $\tau_{\phi}(\mathbb{R})$ is a $G_{+}$-invariant set, because for each $g \in G_{+}$ we have

by Claim 4.1.

$$
g\left(\tau_{\phi}(\mathbb{R})\right)=\tau_{\phi}(g(\mathbb{R}))=\tau_{\phi}(\mathbb{R})
$$

Since $\tau_{\phi}(\mathbb{R})$ is $G_{+}$-invariant and the action of $G_{+}$is minimal (see Remark 4), it follows that $\tau_{\phi}(\mathbb{R})$ is dense in $\mathbb{R}$.

We see that the map $\tau_{\phi}$ is monotone (Claim 4.3), and its image is dense in $\mathbb{R}$. Consequently, by a simple classical argument, the map $\tau_{\phi}$ is continuous.

Now it remains to notice that every continuous map $\mathbb{R} \rightarrow \mathbb{R}$ with dense image is surjective. 
Claim 4.5. The map $\tau_{\phi}$ is injective.

Proof. We define a subset $\mathcal{P}$ of $\mathbb{R}$ as follows. We set $x \in \mathcal{P}$ whenever $x$ has an open neighborhood $B_{x}$ such that the image $\tau_{\phi}\left(B_{x}\right)$ is a singleton. Observe that $\mathcal{P}$ is open because each point $x \in \mathcal{P}$ has an open neighborhood $\left(B_{x}\right)$ in $\mathcal{P}$.

We show that the set $\mathcal{P}$ is $G_{+}$-invariant. Since $G_{+}$is a group, it suffices to prove that $g(\mathcal{P}) \subset \mathcal{P}$ for each $g$ in $G_{+}$. (The conditions $g^{-1}(\mathcal{P}) \subset \mathcal{P}$ and $g(\mathcal{P}) \subset \mathcal{P}$ imply that $\mathcal{P}=g g^{-1}(\mathcal{P}) \subset g(\mathcal{P})$, whence $g(\mathcal{P})=\mathcal{P}$.) Let $x$ be a point in $\mathcal{P}$, and let $B_{x} \subset \mathbb{R}$ be an open neighborhood of $x$ such that $\tau_{\phi}\left(B_{x}\right)$ is a point (say $\tau_{\phi}\left(B_{x}\right)=: z$ ). Then, by Claim 4.1, we have $\tau_{\phi}\left(g\left(B_{x}\right)\right)=g\left(\tau_{\phi}\left(B_{x}\right)\right)=g(z)$, i.e., $\tau_{\phi}$ maps the open neighborhood $g\left(B_{x}\right)$ of the point $g(x)$ to a point. This means that $g(x) \in \mathcal{P}$. Since $x$ is an arbitrary point in $\mathcal{P}$, it follows that $g(\mathcal{P}) \subset \mathcal{P}$. Thus, the set $\mathcal{P}$ is $G_{+}$-invariant.

Since the action of $G_{+}$is minimal (see Remark 4 ), the only open $G_{+}$-invariant subsets in $\mathbb{R}$ are the empty subset and $\mathbb{R}$ itself. Therefore, we have either $\mathcal{P}=\mathbb{R}$ or $\mathcal{P}=\varnothing$. If $\mathcal{P}=\mathbb{R}$, then the connectedness of $\mathbb{R}$ implies that the image $\tau_{\phi}(\mathbb{R})$ is a singleton, which contradicts the surjectivity of $\tau_{\phi}$ (see Claim 4.4). Consequently, $\mathcal{P}$ is empty.

Finally, we use the emptiness of $\mathcal{P}$ to check that $\tau_{\phi}$ is injective. Assume that $\tau_{\phi}$ is not injective. Then there are two points $u<v$ such that $\tau_{\phi}(u)=\tau_{\phi}(v)$. Hence, since $\tau_{\phi}$ is monotone, the image $\tau_{\phi}([u, v])$ of $[u, v]$ is a point. Consequently, $(u, v) \subset \mathcal{P}$ by the definition of $\mathcal{P}$. This contradicts the fact that $\mathcal{P}=\varnothing$. Hence, $\tau_{\phi}$ is injective.

Proof of 2). By Claims 4.3-4.5, the map $\tau_{\phi}: \mathbb{R} \rightarrow \mathbb{R}$ is a continuous bijection. It is well known that this implies (in the case of $\mathbb{R}$ ) that our map is a homeomorphism. By Claim $4.2, \tau_{\phi}$ has no fixed points.

Proof of 3). This follows from Lemma 1.4 and assertion 2) of the proposition.

Proof of 4). By Claim 4.1, the homeomorphism $\tau_{\phi}$ commutes with the action of $G_{+}$. Let $\mathcal{Z}$ be the centralizer of $\phi\left(G_{+}\right)$in $\operatorname{Homeo}(\mathbb{R})$. We show that $\tau_{\phi}$ generates $\mathcal{Z}$. Let $\xi$ be an arbitrary element in $\mathcal{Z}$. We observe that, by Claim 4.2 , the two-sided sequence $\left\{\tau_{\phi}^{i}(0)\right\}_{i \in \mathbb{Z}}$ is monotone increasing: for each $i \in \mathbb{Z}$ we have $\tau_{\phi}^{i}(0)<\tau_{\phi}^{i+1}(0)$. Furthermore, since $\tau_{\phi}$ has no fixed points (see assertion 2), it follows that $\left\{\tau_{\phi}^{i}(0)\right\}_{i \in \mathbb{Z}}$ is unbounded from above and from below. Consequently, there exists an integer $k$ such that $\tau_{\phi}^{k}(0) \leq \xi(0)<\tau_{\phi}^{k+1}(0)$. It follows that

$$
0 \leq \tau_{\phi}^{-k} \xi(0)<\tau_{\phi}(0)
$$

Therefore, the points 0 and $\tau_{\phi}^{-k} \xi(0)$ are $G$-proximal by the definition of $\tau_{\phi}$, and so they are $G_{+}$-proximal (see Remark 4). But the homeomorphism $\tau_{\phi}^{-k} \xi$, being the composition of $\xi$ and $\tau_{\phi}$, commutes with the action of $G_{+}$. Then Lemma 3.4 implies that the map $\tau_{\phi}^{-k} \xi$ is identical, i.e., $\xi=\tau_{\phi}^{k}$. Since $\xi$ is an arbitrary element of $\mathcal{Z}$, it follows that $\tau_{\phi}$ indeed generates $\mathcal{Z}$.

Proof of 5). Since $\phi\left(G_{+}\right)$is a subgroup of index at most 2 in $\phi(G)$, it follows that $\phi\left(G_{+}\right)$ is a normal subgroup of $\phi(G)$. In other words, $\phi(G)$ is contained in the normalizer of $\phi\left(G_{+}\right)$. By Lemma 3.5, this implies that $\phi(G)$ is contained in the normalizer of $\left\langle\tau_{\phi}\right\rangle$. (Recall that, by assertion 4) of the proposition, $\left\langle\tau_{\phi}\right\rangle$ is the centralizer of $\phi\left(G_{+}\right)$.) Then, by Lemma 1.3 , there exists a (unique) action $\psi: G \rightarrow \operatorname{Homeo}\left(\mathbb{R} / \tau_{\phi}\right)$ such that $\phi$ is semiconjugate to $\psi$ via $\mathbf{p}_{\tau_{\phi}}$.

Proof of 6). First, we show that $\psi$ is proximal. Let $x$ and $y$ be two arbitrary points of the circle $\mathbb{R} / \tau_{\phi}$. Since the image of the interval $\left[0, \tau_{\phi}(0)\right)$ under the quotient map $\mathbf{p}_{\tau_{\phi}}: \mathbb{R} \rightarrow \mathbb{R} / \tau_{\phi}$ covers the circle $\mathbb{R} / \tau_{\phi}$, it follows that some points $\tilde{x} \in \mathbf{p}_{\tau_{\phi}}^{-1}(x)$ and $\tilde{y} \in \mathbf{p}_{\tau_{\phi}}^{-1}(y)$ lie in $\left[0, \tau_{\phi}(0)\right)$. The definition of $\tau_{\phi}$ implies that $\tilde{x}$ and $\tilde{y}$ are $G$-proximal, 
i.e., there exists a sequence $\left\{g_{k}\right\}$ in $G$ such that the sequences $\left\{g_{k} \tilde{x}\right\}$ and $\left\{g_{k} \tilde{y}\right\}$ converge to one and the same point $\tilde{z} \in \mathbb{R}$. Therefore, the sequences $\left\{\psi\left(g_{k}\right) x\right\}=\left\{\mathbf{p}_{\tau_{\phi}}\left(g_{k} x\right)\right\}$ and $\left\{\psi\left(g_{k}\right) y\right\}=\left\{\mathbf{p}_{\tau_{\phi}}\left(g_{k} y\right)\right\}$ converge to one and the same point $\mathbf{p}_{\tau_{\phi}}(\tilde{z}) \in \mathbb{R} / \tau_{\phi}$, i.e., $x$ and $y$ are $\psi$-proximal. Since $x$ and $y$ were chosen arbitrarily, $\psi$ is proximal.

The minimality of $\psi$ follows from the minimality of $\phi$, in view of the fact that no minimal action is semiconjugate to a nonminimal action. To prove this, assume that an action $\phi: G \rightarrow \operatorname{Homeo}(\mathcal{X})$ is semiconjugate to a nonminimal action $\psi: G \rightarrow \operatorname{Homeo}(\mathcal{Y})$ via a (continuous and surjective) semiconjugacy $p: \mathcal{X} \rightarrow \mathcal{Y}$. Since $\psi$ is not minimal, there is a nonempty open subset $U \subset \mathcal{Y}$ and a point $y \in \mathcal{Y}$ such that the orbit $G(y)$ does not hit $U$. Then the nonempty open subset $p^{-1}(U) \subset \mathcal{X}$ is disjoint from those orbits of $\phi$ that compose the set $p^{-1}(G(y))$. This means that $\phi$ is not minimal.

\section{§5. Minimal and nondistal group aCtions on the Circle}

Proposition 5. Suppose that an action $\phi: G \rightarrow \operatorname{Homeo}\left(\mathbf{S}^{1}\right)$ of a group $G$ is minimal and nondistal. Then $\phi$ is a cover of a minimal and proximal action on the circle.

Proof. Recall that $\mathbf{p}: \mathbb{R} \rightarrow \mathbf{S}^{1}$ is the universal covering (see Subsection 1.4 for the description of $\mathbf{p})$. Let $\widetilde{G}$ be the subgroup of $\widetilde{\operatorname{Homeo}}\left(\mathbf{S}^{1}\right) \subset \operatorname{Homeo}(\mathbb{R})$ consisting of all lifts of homeomorphisms in $\phi(G)$ :

$$
\widetilde{G}:=\mathbf{p}_{*}^{-1}(\phi(G)) .
$$

For convenient reference, we denote by $\psi$ the injection $\widetilde{G} \rightarrow \operatorname{Homeo}(\mathbb{R})$, i.e., the action of $\widetilde{G}$ on $\mathbb{R}$ :

$$
\psi: \widetilde{G} \rightarrow \operatorname{Homeo}(\mathbb{R}) .
$$

Claim 5.1. The action $\psi$ is minimal and neither distal nor proximal.

Proof. The action $\psi$ is not proximal because $\psi(\widetilde{G})=\widetilde{G} \subset \mathrm{Homeo}\left(\mathbf{S}^{1}\right)$.

The fact that $\psi$ is not distal follows from Lemma 2.1 (recall the definition of $\widetilde{G}$ and observe that the action of the group $\phi(G) \subset \operatorname{Homeo}\left(\mathbf{S}^{1}\right)$ on $\mathbf{S}^{1}$ is not distal because $\phi$ is not distal).

It remains to show that $\psi$ is minimal, which means by definition that for each point $x \in \mathbb{R}$ the orbit $\widetilde{G}(x)$ is dense in $\mathbb{R}$. Since $\widetilde{G}$ contains the translation by 1 (which is a lift of the identity homeomorphism), it follows that the orbit $\widetilde{G}(x)$ coincides with the inverse image $\mathbf{p}^{-1}(G(\mathbf{p}(x)))$ of the orbit $G(\mathbf{p}(x))$ of the action $\phi$. Since $\phi$ is minimal, the orbit $G(\mathbf{p}(x))$ is dense in $\mathbf{S}^{1}$. We also observe that $\mathbf{p}$ is open, being a covering map. Clearly, the inverse image of a dense set under an open map is a dense set. So $\psi$ is minimal.

Claim 5.1 and Proposition 4 imply the following result.

Claim 5.2. There exists a homeomorphism $\tau \in \operatorname{Homeo}(\mathbb{R})$ such that the following conditions are fulfilled.

- The group $\langle\tau\rangle$ generated by $\tau$ is the centralizer of the subgroup $\widetilde{G}_{+}:=\widetilde{G} \cap$ $\mathrm{Homeo}_{+}(\mathbb{R})$ in $\operatorname{Homeo}(\mathbb{R})$.

- The orbit space $\mathbb{R} / \tau$ is homeomorphic to the circle. The corresponding quotient $\operatorname{map} \mathbf{p}_{\tau}: \mathbb{R} \rightarrow \mathbb{R} / \tau$ is a universal covering map.

- The action $\psi$ is semiconjugate via $\mathbf{p}_{\tau}$ to an action $\psi_{\tau}: \widetilde{G} \rightarrow \operatorname{Homeo}(\mathbb{R} / \tau)$.

- The action $\psi_{\tau}$ is minimal and proximal.

Now, we observe that since $\widetilde{G}$ is a subgroup of $\widetilde{H o m e o}\left(\mathbf{S}^{1}\right)$, the subgroup $\widetilde{G}_{+}$is con-

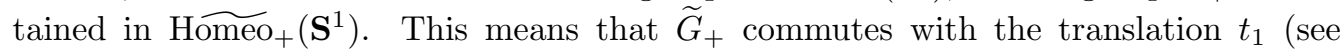
Subsection 1.4). In other words, the subgroup $\left\langle t_{1}\right\rangle$ of integer translations is contained 
in the centralizer of $\widetilde{G}_{+}$. By Claim 5.2, this centralizer coincides with the subgroup $\langle\tau\rangle$. Consequently, the translation $t_{1}$ is a power of $\tau$ (say $t_{1}=\tau^{k}$, where $k \in \mathbb{Z}$ ). By Lemma 1.4, there exists a conjugacy $h: \mathbb{R} \rightarrow \mathbb{R}$ such that $h \tau h^{-1}$ is the translation $t_{1}$. We see that $h t_{1} h^{-1}=h \tau^{k} h^{-1}=t_{1}^{k}=t_{k}$. Since the circles $\mathbf{S}^{1}$ and $\mathbb{R} / \tau$ are the orbit spaces of the actions $\left\langle t_{1}\right\rangle$ and $\langle\tau\rangle$, respectively, we obtain the following claim.

Claim 5.3. Let

$$
\mathbf{q}: \mathbf{S}^{1} \rightarrow \mathbb{R} / \tau
$$

be the map that takes the orbit $\left\langle t_{1}\right\rangle(x) \in \mathbf{S}^{1}$ to the orbit $\langle\tau\rangle(x) \in \mathbb{R} / \tau$ for each $x \in \mathbb{R}$. Then $\mathbf{q}$ is a $|k|$-sheeted covering. Furthermore, we have

$$
\mathbf{p}_{\tau}=\mathbf{q} \circ \mathbf{p},
$$

where $\mathbf{p}: \mathbb{R} \rightarrow \mathbf{S}^{1}$ is the standard universal covering, while $\mathbf{p}_{\tau}: \mathbb{R} \rightarrow \mathbb{R} / \tau$ is the universal covering defined in Claim 5.2.

Now, we consider a natural action of $\widetilde{G}$ on $\mathbf{S}^{1}$, which we denote by $\psi_{1}$ :

$$
\psi_{1}:=\mathbf{p}_{*} \circ \psi: \widetilde{G} \rightarrow \operatorname{Homeo}\left(\mathbf{S}^{1}\right) .
$$

By definition, $\psi$ is semiconjugate to $\psi_{1}$ via the covering $\mathbf{p}$. We also observe that the above definitions imply that

$$
\psi_{1}(\widetilde{G})=\mathbf{p}_{*}(\widetilde{G})=\phi(G) .
$$

Claim 5.4. The action $\psi_{1}: \widetilde{G} \rightarrow \operatorname{Homeo}\left(\mathbf{S}^{1}\right)$ is semiconjugate to the action $\psi_{\tau}: \widetilde{G} \rightarrow$ $\operatorname{Homeo}(\mathbb{R} / \tau)$ via the covering $\mathbf{q}$.

Proof. Let $g$ be an arbitrary element of $\widetilde{G}$, let $x$ be an arbitrary point in $\mathbf{S}^{1}$, and let $\tilde{x} \in \mathbf{p}^{-1}(x)$ be a point of the inverse image $\mathbf{p}^{-1}(x) \subset \mathbb{R}$. Then the identity $\mathbf{p}_{\tau}=\mathbf{q} \circ \mathbf{p}$ of Claim 5.3 and the fact that $\psi$ is semiconjugate to $\psi_{1}$ via $\mathbf{p}$ imply that

$$
\mathbf{q}\left(\psi_{1} g(x)\right)=\mathbf{q}\left(\psi_{1} g(\mathbf{p} \tilde{x})\right)=\mathbf{q}(\mathbf{p}(g(\tilde{x})))=\mathbf{p}_{\tau}(g(\tilde{x})) .
$$

On the other hand, since $\psi$ is semiconjugate to $\psi_{\tau}$ via $\mathbf{p}_{\tau}$, we have

$$
\psi_{\tau} g(\mathbf{q}(x))=\psi_{\tau} g\left(\mathbf{p}_{\tau}(\tilde{x})\right)=\mathbf{p}_{\tau}(g(\tilde{x})) .
$$

Therefore,

$$
\psi_{\tau} g(\mathbf{q}(x))=\mathbf{p}_{\tau}(g(\tilde{x}))=\mathbf{q}\left(\psi_{1} g(x)\right) .
$$

This means precisely that $\psi_{1}$ is semiconjugate to $\psi_{\tau}$ via $\mathbf{q}$.

Claim 5.4 and the above-mentioned relation $\phi(G)=\psi_{1}(\widetilde{G})$ easily imply that $\phi$ is semiconjugate via $\mathbf{q}$ to a certain action $\phi_{2}: G \rightarrow \operatorname{Homeo}(\mathbb{R} / \tau)$. Obviously, we have $\phi_{2}(G)=\psi_{2}(\widetilde{G})$, whence it follows that $\phi_{2}$ is minimal and proximal because so is $\psi_{2}$.

We have shown that $\phi$ is semiconjugate via the covering $\mathbf{q}$ to the minimal and proximal action $\phi_{2}$ on the circle $\mathbb{R} / \tau$. The proposition is proved.

\section{§6. Minimal and proximal group actions on the Circle}

Proposition 6. Suppose that an action $\phi: G \rightarrow \operatorname{Homeo}\left(\mathbf{S}^{1}\right)$ of a group $G$ on the circle is minimal and proximal. Then for each proper closed subset $V \subset \mathbf{S}^{1}$ and each nonempty open subset $U \subset \mathbf{S}^{1}$ there exists an element $g \in G$ such that $g(V) \subset U$.

Remark 6.1. Obviously, Proposition 6 implies that no minimal and proximal action $\phi$ : $G \rightarrow \operatorname{Homeo}\left(\mathbf{S}^{1}\right)$ admits a $\phi$-invariant Borel probability measure on $\mathbf{S}^{1}$. 
Remark 6.2. Proposition 6 also implies that a group $G$ contains a free non-Abelian subgroup whenever there exists a minimal and proximal action $\phi: G \rightarrow \operatorname{Homeo}\left(\mathbf{S}^{1}\right)$. To prove this, consider a quadruple $U_{1}, U_{2}, U_{3}, U_{4}$ of proper open pairwise disjoint subsets of $\mathbf{S}^{1}$. By Proposition 6, $G$ contains two elements $g$ and $h$ such that $g\left(X \backslash U_{1}\right) \subset U_{2}$ and $h\left(X \backslash U_{3}\right) \subset U_{4}$. Since $g\left(U_{2}\right) \subsetneq U_{2}$ and $h\left(U_{4}\right) \subsetneq U_{4}$, it follows that both $g$ and $h$ have infinite order. We set $A:=U_{1} \cup U_{2}$ and $B:=U_{3} \cup U_{4}$. Then we have $g^{ \pm 1}(B) \subset A$ and $h^{ \pm 1}(A) \subset B$. It easily follows that the homeomorphisms $\phi(g)$ and $\phi(h)$ generate a free non-Abelian subgroup in $\operatorname{Homeo}\left(\mathbf{S}^{1}\right)$ (see, e.g., [5, Chapter III, §12] for a detailed proof).

Proof of Proposition 6. For an ordered pair $(x, y)$ of distinct points on the circle, we define a closed interval $[x, y]$ in $\mathbf{S}^{1}$ as follows. Let $\widetilde{x}$ be an arbitrary point of the inverse image $\mathbf{p}^{-1}(x)$ (we recall that $\mathbf{p}: \mathbb{R} \rightarrow \mathbb{R} / t_{1}=\mathbf{S}^{1}$ is the universal covering), and let $\widetilde{y}$ be the only point in the singleton $\mathbf{p}^{-1}(y) \cap(\widetilde{x}, \widetilde{x}+1)$. We set $[x, y]:=\mathbf{p}([\widetilde{x}, \widetilde{y}])$. (Clearly, $\mathbf{p}([\widetilde{x}, \widetilde{y}])$ does not depend on the choice of $\widetilde{x}$. $)$

We consider the space $L:=\mathbf{S}^{1} \times \mathbf{S}^{1} \backslash \Delta$ of all ordered pairs of distinct points of the circle. (Here, as is customary, $\Delta$ denotes the diagonal $\left\{(x, x) \mid x \in \mathbf{S}^{1}\right\} \subset \mathbf{S}^{1} \times \mathbf{S}^{1}$.) Let $i: L \rightarrow L$ be the involution that sends a pair $(x, y)$ to the pair $(y, x)$.

We define

$$
\Omega:=\{(x, y) \in L:[x, y] \text { is } G \text {-contractible }\} .
$$

Observe that $\Omega$ is an open subset of $L$. (To prove this, consider an arbitrary point $(x, y) \in \Omega$. By definition, the interval $[x, y]$ is $G$-contractible. Furthermore, since $\phi$ is minimal, $G$ contains an element $g$ such that $g([x, y]) \subset \operatorname{int}([x, y])$. The rest is easy.)

It is obvious that if two distinct points $u \neq v \in \mathbf{S}^{1}$ are $G$-proximal, then at least one of the intervals $[u, v]$ and $[v, u]$ is $G$-contractible. Then the proximality of $\phi$ implies that $\Omega \cup i(\Omega)=L$. Since $L$ is connected, $\Omega$ is open, and $i$ is a homeomorphism, it follows that the intersection $\Omega \cap i(\Omega)$ is nonempty. In other words, there exists a pair $(a, b) \in L$ such that both intervals $[a, b]$ and $[b, a]$ are $G$-contractible.

Now, we show that for any two distinct points $x \neq y \in \mathbf{S}^{1}$, the interval $[x, y]$ is $G$ contractible. (In other words, $L=\Omega$.) Since $[a, b]$ is $G$-contractible and $\phi$ is minimal, we see that there exists an element $g \in G$ such that $g([a, b]) \subset[y, x]$. Then $g([b, a]) \supset[x, y]$. The interval $g([b, a])$ is $G$-contractible because so is $[b, a]$. Since $g([b, a])$ contains $[x, y]$, it follows that $[x, y]$ is also $G$-contractible.

To complete the proof, we notice that each proper closed subset of the circle is contained in some nondegenerate closed interval. Then it follows that every such subset is $G$-contractible. Now, the required statement follows from the minimality of the action under consideration.

\section{$\S 7$. Proofs of Theorem 1 and Corollary 2}

Here we prove our main Theorem 1 and Corollary 2 .

Theorem 1. Let $X$ be either the real line $\mathbb{R}$ or the circle $\mathbf{S}^{1}$. Let $\phi: G \rightarrow \operatorname{Homeo}(X)$ be a minimal action of a group $G$ on $X$ (by homeomorphisms). Then exactly one of the following three assertions $(i)-(i i i)$ is true:

(i) the action $\phi$ is conjugate to an isometric action;

(ii) the action $\phi$ is proximal;

(iii) the action $\phi$ is a nontrivial cover of a minimal and proximal action on the circle, i.e., $\phi$ is semiconjugate to a minimal and proximal action on the circle via a nontrivial covering map. 
Proof. It is obvious that the conditions of proximality and distality are mutually exclusive whenever the space on which our group acts has at least two points. Therefore, the theorem will be proved if we check the following three claims.

1) Assertion (i) is true if and only if $\phi$ is distal.

2) Assertion (ii) is true if and only if $\phi$ is proximal.

3) Assertion (iii) is true if and only if $\phi$ is neither distal nor proximal.

If $\phi$ is distal, then $\phi$ is conjugate to an isometric action by Proposition 2. On the other hand, each isometric action is distal by definition. Hence, each action conjugate to an isometric action is also distal. Therefore, Claim 1) is true. Claim 2) is trivial, because assertion (ii) says that $\phi$ is proximal. Claim 3) immediately follows from Claims 7.1-7.3 presented below.

Claim 7.1. If $\phi$ is neither distal nor proximal, then assertion (iii) is true.

Proof. If $X=\mathbb{R}$, then $\phi$ meets the assumptions of Proposition 4 , while if $X=\mathbf{S}^{1}$, then $\phi$ meets the assumptions of Proposition 5. By these propositions, $\phi$ is a cover of a minimal and proximal action on the circle. Moreover, the covering is nontrivial, because otherwise $\phi$ would be proximal, contrary to our assumptions.

Claim 7.2. If assertion (iii) is true, then $\phi$ is not proximal.

Proof. By our definitions, if assertion (iii) is true, then $\phi$ is semiconjugate to an action on the circle via a nontrivial covering $p: X \rightarrow \mathbf{S}^{1}$. Since $p$ is nontrivial, the inverse image $p^{-1}(x)$ of a point $x \in \mathbf{S}^{1}$ contains at least two distinct points $x_{1} \neq x_{2} \in X$. We see at once that the points $x_{1}$ and $x_{2}$ are $G$-distal. Hence, $\phi$ is not proximal.

Claim 7.3. If assertion (iii) is true, then $\phi$ is not distal.

Proof. We use the notion of amenability. Recall that a group $\Gamma$ endowed with the discrete topology is amenable if and only if for each action $\psi: \Gamma \rightarrow \operatorname{Homeo}(\mathcal{X})$ on any compact nonempty topological space $\mathcal{X}$ there is a $\Gamma$-invariant Borel probability measure on $\mathcal{X}$. It is known that all Abelian groups are amenable, and an extension of an amenable group by an amenable group is an amenable group.

To prove our claim, it obviously suffices to check the following two assertions.

1) If assertion (iii) is true, then the group $\phi(G)$ is not amenable.

2) If an action $\phi: G \rightarrow \operatorname{Homeo}(X)$ is distal, then the group $\phi(G)$ is amenable.

1) If (iii) is true, then the action $\phi: G \rightarrow \operatorname{Homeo}(X)$ is semiconjugate to a minimal and proximal action $\psi: G \rightarrow \operatorname{Homeo}\left(\mathbf{S}^{1}\right)$. Hence, there exists an (obviously, unique) homomorphism (an action) $\xi: \phi(G) \rightarrow \operatorname{Homeo}\left(\mathbf{S}^{1}\right)$ such that $\psi=\xi \circ \phi$. However, since $\xi(\phi(G))=\psi(G)$, it follows that $\xi$ is minimal and proximal (because so is $\psi$ ). Hence by Proposition 6 it follows that $\mathbf{S}^{1}$ bears no $\xi$-invariant Borel probability measures (see Remark 6.1). Then the above-mentioned property of amenable groups implies that $\phi(G)$ is not amenable.

2) If $\phi$ is distal, then, by Proposition 2, the group $\phi(G)$ is conjugate in $\operatorname{Homeo}(X)$ to a subgroup consisting of isometries of $X$. Consequently, the group $\phi(G) \cap \mathrm{Homeo}_{+}(X)$ is conjugate to a subgroup consisting of orientation-preserving isometries of $X$. Clearly, this means that $\phi(G) \cap \mathrm{Homeo}_{+}(X)$ is Abelian. Since $\phi(G) \cap \mathrm{Homeo}_{+}(X)$ is a subgroup of index at most 2 in $\phi(G)$, the aforementioned properties of amenable groups (see the beginning of the proof of Claim 7.3) imply that $\phi(G)$ is amenable.

Corollary $2\left(\mathbf{S}^{1}\right)$. Let $\phi: G \rightarrow$ Homeo $\left(\mathbf{S}^{1}\right)$ be an action of a group $G$ on the circle (by homeomorphisms). Then exactly one of the following three conditions is fulfilled:

(a) the action $\phi$ has a finite orbit; 
(b) the action $\phi$ is semiconjugate to an isometric minimal action on the circle via a continuous locally monotone semiconjugacy of degree 1 ;

(c) the action $\phi$ is semiconjugate to a minimal and proximal action on the circle via a continuous locally monotone semiconjugacy.

Proof. It is well known that an action of a group $G$ on the circle by homeomorphisms either has a finite orbit, or is minimal, or has a (unique) minimal $G$-invariant subset $K$ that is perfect and nowhere dense (see, e.g., $[2,1]$ ). In the third case, the action is semiconjugate to a minimal action on the circle via a locally monotone map of degree 1 , which maps the closure of an interval of $\mathbf{S}^{1} \backslash K$ to a point (see $[2,1]$ ). This implies the following lemma.

Lemma 7.1. An action of a group on the circle by homeomorphisms either has a finite orbit, or is semiconjugate to a minimal action on the circle via a continuous locally monotone semiconjugacy of degree 1.

Next, we observe that each covering $\mathbf{S}^{1} \rightarrow \mathbf{S}^{1}$ is locally monotone, and that a composition of locally monotone maps is also locally monotone. Combining these facts with Lemma 7.1 and Theorem 1, we see that an arbitrary action $\phi: G \rightarrow \operatorname{Homeo}\left(\mathbf{S}^{1}\right)$ satisfies at least one of conditions (a), (b), and (c).

It remains to show that conditions (a)-(c) are pairwise incompatible. Condition (a) is compatible neither with (b) nor with (c) because no action with a finite orbit is semiconjugate to a minimal action on the circle. Conditions (b) and (c) are incompatible by the following claim.

Claim 7.4. Condition (b) implies that there is a $\phi(G)$-invariant Borel probability measure on the circle, while condition (c) implies that such a measure cannot exist.

Proof. 1) Suppose that condition (b) is fulfilled, so that the action $\phi: G \rightarrow \operatorname{Homeo}\left(\mathbf{S}^{1}\right)$ is semiconjugate to an isometric action $\psi: G \rightarrow \operatorname{Homeo}\left(\mathbf{S}^{1}\right)$ via a locally monotone continuous map $f: \mathbf{S}^{1} \rightarrow \mathbf{S}^{1}$ of degree 1 . We see that, by definition, the Lebesgue measure $\lambda$ is a $\psi(G)$-invariant Borel probability measure on $\mathbf{S}^{1}$. Since $f$ is locally monotone and has degree 1 , the inverse image $f^{-1}(x)$ of a point $x \in \mathbf{S}^{1}$ is either a point or a nondegenerate closed interval. Let $P$ be the subset of $\mathbf{S}^{1}$ defined as follows: $x \in$ $P$ if $f^{-1}(x)$ is a point. Then the restriction $\left.f^{-1}\right|_{P}$ is a bijection from $P$ to $f^{-1}(P)$. Furthermore, the set $\mathbf{S}^{1} \backslash P$ is at most countable, whence $\lambda\left(\mathbf{S}^{1} \backslash P\right)=0$. Consequently, the measure $\left.f^{-1}\right|_{P}(\lambda)$ is a $\phi(G)$-invariant Borel probability measure on the circle.

2) Now, assume condition (c). Assume that $\mathbf{S}^{1}$ bears a $\phi(G)$-invariant Borel probability measure $\mu$. By condition (c), the action $\phi: G \rightarrow \operatorname{Homeo}\left(\mathbf{S}^{1}\right)$ is semiconjugate to a minimal and proximal action $\psi: G \rightarrow \operatorname{Homeo}\left(\mathbf{S}^{1}\right)$ via a continuous locally monotone semiconjugacy $f: \mathbf{S}^{1} \rightarrow \mathbf{S}^{1}$. Then it is easily seen that the measure $f(\mu)$ is a $\psi(G)$-invariant Borel probability measure, which is impossible by Proposition 6 (see Remark 6.1). This is a contradiction, and our claim is proved.

Acknowledgements. The author is grateful to A. M. Vershik and N. Yu. Netsvetaev for useful discussions. Thanks for valuable suggestions are also due to V. A. Kaimanovich, who told the author about the Ghys-Margulis alternative.

\section{REFERENCES}

[1] L. A. Beklaryan, Groups of homeomorphisms of the line and the circle. Topological characteristics and metric invariants, Uspekhi Mat. Nauk 59 (2004), no. 4, 3-68; English transl., Russian Math. Surveys 59 (2004), no. 4, 599-660. MR2106645 (2005i:37044)

[2] É. Ghys, Groups acting on the circle, Enseign. Math. (2) 47 (2001), 329-407. MR1876932 (2003a:37032) 
[3] H. Furstenberg, Boundary theory and stochastic processes on homogeneous spaces, Harmonic Analysis on Homogeneous Spaces (Williams College, Williamstown, MA, 1972), Proc. Sympos. Pure Math., vol. 26, Amer. Math. Soc., Providence, RI, 1973, pp. 193-229. MR0352328 (50:4815)

[4] A. Katok and B. Hasselblatt, Introduction to the modern theory of dynamical systems, Encyclopedia Math. Appl., vol. 54, Cambridge Univ. Press, Cambridge, 1995. MR1326374 (96c:58055)

[5] R. C. Lyndon and P. E. Schupp, Combinatorial group theory, Ergeb. Math. Grenzgeb., Bd. 89, Springer-Verlag, Berlin-New York, 1977. MR0577064 (58:28182)

[6] G. A. Margulis, Free subgroups of the homeomorphism group of the circle, C. R. Acad. Sci. Paris Sér. I Math. 331 (2000), no. 9, 669-674. MR1797749 (2002b:37034)

St. Petersburg Branch, Steklov Mathematical Institute, Russian Academy of Sciences, Fontanka 27, St. Petersburg 191023, Russia

E-mail address: malyutin@pdmi.ras.ru

Received 16/JUN/2006

Translated by THE AUTHOR 